Reprinted from American Journal of Physics, Vol. 26, No. 7, 467-469, October, 1958

Printed in U. S. A.

\title{
Elementary Derivation of the Multicomponent Diffusion Equation*
}

\author{
F. A. WiLliams $\dagger$ \\ Daniel and Florence Guggenheim Jet Propulsion Center, California Institute of Technology, \\ Pasadena, California
}

(Received February 13, 1958)

\begin{abstract}
It is demonstrated that the multicomponent diffusion equation can be derived from elementary kinetic-theory considerations without employing the results of the mathematical theory of nonuniform gases. By using the approach described by Furry, elementary expressions for the binary diffusion coefficients are also obtained. The advantage of the concept of the equivalence of partial pressure gradients and momentum gradients is thereby emphasized.
\end{abstract}

$I^{\mathrm{T}}$ $T$ has been emphasized by Furry ${ }^{1}$ that the usual elementary treatments of transport processes, which involve the concept of mean free path, lead to results which are qualitatively incorrect for diffusion. It has been demonstrated that if one uses the alternate viewpoint of calculating the diffusion velocities necessary to support specified concentration gradients, then the results are in quantitative agreement with results derived from the accurate kinetic theory of gases. In this note we use the approach suggested by Furry to derive the multicomponent diffusion equation from elementary considerations. Thermal diffusion is neglected, but the effects of pressure gradients and body forces are included. We also obtain approximate expressions for the binary diffusion coefficients.

The total momentum is conserved in molecular collisions. Hence the momentum of molecules of type $i$ can be changed only by collisions with molecules of other types $(j \neq i)$ and by body forces acting on species $i$. If $\mathrm{V}_{j}$ is the diffusion velocity of component $j$ and $\mu_{i j}$ is the reduced mass of the colliding molecules, then the average momentum transferred in a collision is, approximately, $\mu_{i j}\left(\mathrm{~V}_{j}-\mathrm{V}_{i}\right)$, where $\left(\mathrm{V}_{j}-\mathrm{V}_{i}\right)$ is seen to be the average relative velocity of species $i$ and $j$. The body force acting on the molecules of type $i$ in a unit volume element is given by $\rho Y_{i} \mathbf{F}_{i}$, where $\rho$ is the fluid density, $Y_{i}$ represents the mass fraction of component $i$, and $\mathbf{F}_{i}$ is the body force on a molecule of species $i$ per unit molec-

* Supported in part by the Office of Ordnance Research, U. S. Army, under Contract DA-04-495-Ord-446.

$\dagger$ National Science Foundation predoctoral fellow.

I W. H. Furry, Am. J. Phys. 16, 63-78 (1948). References to earlier work on this subject may be found in the article by Furry. ular mass. If the symbol $\boldsymbol{\Gamma}_{i}$ is used to denote the net rate of change of momentum of molecules of type $i$ per unit volume, then

$$
\boldsymbol{\Gamma}_{i}=\sum_{i=1}^{s} \mu_{i j} \nu_{i j}\left(\mathrm{~V}_{j}-\mathrm{V}_{i}\right)+\rho Y_{i} \mathbf{F}_{i},
$$

where $\nu_{i j}$ is the collision frequency and $s$ denotes the total number of chemical components.

The change in momentum of molecules of species $i$ includes changes in the random velocity and in the ordered velocity. The rate of change of the ordered momentum is $\rho Y_{i} D \mathrm{v} / D t$, where $\mathbf{v}$ is the mass average velocity of the fluid and $D / D t$ represents the substantial derivative with respect to time. Since the partial pressure $p_{i}$ of species $i$ is the momentum of random motion of molecules of type $i$ transported per unit time across a unit surface area traveling with the mass average velocity of the fluid, ${ }^{2}$ it follows that the gradient of $p_{i}\left(\nabla p_{i}\right)$ is the rate of change of the momentum of random motion of molecules of type $i$ per unit volume. The quantity $\boldsymbol{\Gamma}_{i}$ is therefore also given by the relation

$$
\Gamma_{i}=\rho Y_{i} \frac{D \mathbf{v}}{D t}+\nabla p_{i}
$$

Since the partial pressure of species $i$ is related to the mole fraction $X_{i}$ of species $i$ and the total hydrostatic pressure $p$ by the expression

$$
p_{i}=X_{i} p
$$

${ }^{2}$ See, for example, J. Jeans, The Dynamical Theory of Gases (Cambridge University Press, London, 1925), Sec. 146, pp. 115-117; or G. N. Patterson, Molecular Flow of Gases (John Wiley and Sons, Inc., New York, 1956), Sec. 3.6, pp. 81-86. 
Eq. (2) may be written in the form

$$
\dot{\boldsymbol{\Gamma}_{i}}=\rho Y_{i} \frac{D \mathbf{v}}{D t}+p \boldsymbol{\nabla} X_{i}+X_{i} \boldsymbol{\nabla} p
$$

To the order of approximation used here the nondiagonal elements of the pressure tensor are negligible. ${ }^{3}$ The conservation of total momentum is therefore given by the equation

$$
\frac{D \mathbf{v}}{D t}=-\nabla p+\rho \sum_{j=1}^{s} Y_{j} \mathbf{F}_{j}
$$

If this relation is substituted into Eq. (4), then

$$
\boldsymbol{\Gamma}_{i}=p \nabla X_{i}+\left(X_{i}-Y_{i}\right) \nabla p+\rho Y_{i} \sum_{j=1}^{s} Y_{j} \mathbf{F}_{j} .
$$

The desired diffusion equation is obtained by equating the values for $\boldsymbol{\Gamma}_{\boldsymbol{i}}$ derived in Eqs. (1) and (5), viz.,

$$
\begin{aligned}
\nabla X_{i}=\sum_{j=1}^{s} \frac{\mu_{i j} \nu_{i j}}{p}\left(\mathrm{~V}_{j}-\mathrm{V}_{i}\right) & +\left(Y_{i}-X_{i}\right) \frac{\boldsymbol{\nabla} p}{p} \\
& +\frac{\rho}{p} \sum_{j=1}^{s} Y_{i} Y_{j}\left(\mathrm{~F}_{i}-\mathrm{F}_{j}\right)
\end{aligned}
$$

where use has been made of the identity

$$
\sum_{j=1}^{8} Y_{j}=1
$$

The meaning of the product $\mu_{i j} \nu_{i j}$ may be found by considering the limiting case of a constant pressure process in a two-component system with no body forces. If we denote the two components by the subscripts 1 and 2, then Eq. (6) becomes

$$
\boldsymbol{\nabla} X_{1}=\frac{\mu_{12} \nu_{12}}{p}\left(\mathrm{~V}_{2}-\mathrm{V}_{1}\right)
$$

The diffusion velocity $V_{1}{ }^{\prime}$ of species 1 with respect to the number-weighted average velocity of the mixture, $\left(\mathrm{v}+\mathrm{V}_{1}\right) X_{1}+\left(\mathrm{v}+\mathrm{V}_{2}\right) X_{2}$, is given by

$$
\mathrm{V}_{1}^{\prime} \equiv \mathrm{v}+\mathrm{V}_{1}-\left(\mathrm{v}+\mathrm{V}_{1}\right) X_{1}-\left(\mathrm{v}+\mathrm{V}_{2}\right) X_{2} .
$$

${ }^{3}$ See, for example, Hirschfelder, Curtiss, and Bird, Molecular Theory of Gases and Liquids (John Wiley and Sons, Inc., New York, 1954), Sec. 7.3c, pp. 468-469.
By using in this relation the identity $\left(1-X_{1}\right)$ $=X_{2}$, we obtain the expression

$$
\mathrm{V}_{1}^{\prime}=X_{2}\left(\mathrm{~V}_{1}-\mathrm{V}_{2}\right)
$$

The substitution of Eq. (8) into Eq. (7) leads to the result

$$
\nabla X_{1}=-\frac{\mu_{12} \nu_{12}}{X_{2} p} \mathrm{~V}_{1}^{\prime}
$$

The binary diffusion coefficient $D_{12}$ for species 1 and 2 is often defined by the equation

$$
D_{12} \nabla X_{1}=-X_{1} \mathrm{~V}^{\prime}{ }^{\prime} \text {. }
$$

It is seen by comparing Eqs. (9) and (10) that

$$
D_{12}=X_{1} X_{2} p / \mu_{12} \nu_{12}
$$

which implies that, in general,

$$
D_{i j}=X_{i} X_{j p} / \mu_{i j} \nu_{i j} \text {. }
$$

An approximate expression for the binary diffusion coefficient $D_{i j}$ can be obtained from Eq. (11). It is readily seen from elementary kinetic theory considerations ${ }^{4}$ that the collision frequency $\nu_{i j}$ is given by the relation

$$
\nu_{i j}=X_{i} X_{j} n^{2} \sigma_{i j} c_{i j},
$$

where $\sigma_{i j}$ represents the collision cross section for molecules of types $i$ and $j$, the quantity $n$ is the total number of molecules per unit volume, and $c_{i j}$ signifies the magnitude of the average relative velocity of the colliding molecules. If Eq. (12) and the ideal gas law,

$$
p=n k T,
$$

are substituted into Eq. (11), then the resulting equation for the diffusion coefficient is

$$
D_{i j}=\frac{k T}{n \mu_{i j} \sigma_{i j} c_{i j}} .
$$

Here $T$ is the temperature and $k$ represents the Boltzmann universal gas constant. The corresponding equation derived from the exact kinetic theory of gases ${ }^{5}$ differs from this prediction only by a constant factor of approximately $\frac{3}{4}$.

\footnotetext{
${ }^{4}$ See, for example, J. Jeans, The Dynamical Theory of Gases (Cambridge University Press, London, 1925), Sec. 33, pp. 35-37.

'See, for example, S. Chapman and T. G. Cowling, The Mathematical Theory of Non-Uniform Gases (Cambridge University Press, London, 1952), Sec. 9.81, p. 165.
} 
By substituting Eq. (11) into Eq. (6) we It can be shown by the exact kinetic theory of obtain the relation gases that this diffusion equation is valid to first

$\nabla X_{i}=\sum_{j=1}^{s} \frac{X_{i} X_{j}}{D_{i j}}\left(\mathrm{~V}_{j}-\mathrm{V}_{i}\right)+\left(Y_{i}-X_{i}\right) \frac{\nabla p}{p}$ order in a Sonine polynomial expansion. ${ }^{6}$ This exercise demonstrates the obvious usefulness and great advantage of the point of view discussed by Furry. ${ }^{1}$

$$
+\frac{\rho}{p} \sum_{j=1}^{s} Y_{i} Y_{j}\left(\mathbf{F}_{i}-\mathbf{F}_{j}\right)
$$

${ }^{6}$ See, for example, Hirschfelder, Curtiss, and Bird, Molecular Theory of Gases and Liquids (John Wiley and Sons, Inc., New York, 1954), Sec. 7.4 ei, pp. 485-488. 